\title{
Global Existence, Uniqueness, and Asymptotic Behavior of Solution for $p$-Laplacian Type Wave Equation
}

\section{Caisheng Chen, Huaping Yao, and Ling Shao}

Department of Mathematics, Hohai University, Nanjing, Jiangsu, 210098, China

Correspondence should be addressed to Caisheng Chen, cshengchen@hhu.edu.cn

Received 10 May 2010; Accepted 13 July 2010

Academic Editor: Michel C. Chipot

Copyright (c) 2010 Caisheng Chen et al. This is an open access article distributed under the Creative Commons Attribution License, which permits unrestricted use, distribution, and reproduction in any medium, provided the original work is properly cited.

We study the global existence and uniqueness of a solution to an initial boundary value problem for the nonlinear wave equation with the $p$-Laplacian operator $u_{t t}-\operatorname{div}\left(|\nabla u|^{p-2} \nabla u\right)-\Delta u_{t}+g(x, u)=$ $f(x)$. Further, the asymptotic behavior of solution is established. The nonlinear term $g$ likes $g(x, u)=a(x)|u|^{\alpha-1} u-b(x)|u|^{\beta-1} u$ with appropriate functions $a(x)$ and $b(x)$, where $\alpha>\beta \geq 1$.

\section{Introduction}

This paper is concerned with the global existence, uniqueness, and asymptotic behavior of solution for the nonlinear wave equation with the $p$-Laplacian operator

$$
\begin{gathered}
u_{t t}-\operatorname{div}\left(|\nabla u|^{p-2} \nabla u\right)-\Delta u_{t}+g(x, u)=f(x), \quad \text { in } \Omega \times(0, \infty), \\
u(x, 0)=u_{0}(x), \quad u_{t}(x, 0)=u_{1}(x), \quad \text { in } \Omega ; \quad u(x, t)=0, \quad \text { on } \partial \Omega \times[0, \infty),
\end{gathered}
$$

where $2 \leq p<n$ and $\Omega$ is a boundary domain in $\mathbf{R}^{n}$ with smooth boundary $\partial \Omega$. The assumptions on $f, g, u_{0}$ and $u_{1}$ will be made in the sequel.

Recently, Ma and Soriano in [1] investigated the global existence of solution $u(t)$ for the problem (1.1)-(1.2) under the assumptions

$$
p=n, \quad g(u) u \geq 0, \quad|g(u)| \leq C_{\beta} \exp \left(\beta|u|^{n /(n-1)}\right), \quad u \in \mathbf{R} .
$$


Moreover, if $f=0$ and $u g(u) \geq G(u)$, then there exist positive constants $c$ and $\gamma$ such that

$$
\begin{gathered}
E(t) \leq c \exp (-\gamma t), \quad t \geq 0, \text { if } n=2, \\
E(t) \leq c(1+t)^{-n /(n-2),} \quad t \geq 0, \text { if } n \geq 3,
\end{gathered}
$$

where

$$
E(t)=\frac{1}{2}\left\|u_{t}(t)\right\|_{2}^{2}+\frac{1}{n}\|\nabla u(t)\|_{n}^{n}+\int_{\Omega} G(x, u(t)) d x
$$

with $G(x, u)=\int_{0}^{u} f(x, s) d s$.

Gao and Ma in [2] also considered the global existence of solution for (1.1)-(1.2). In Theorem 3.1 of [2], the similar results to (1.4)-(1.5) for asymptotic behavior of solution were obtained if the nonlinear function $g(x, u)=g(u)$ satisfies

$$
|g(u)| \leq a|u|^{\sigma-1}+b, \quad u g(u) \geq \rho G(u) \geq 0, \quad \text { in } \Omega \times \mathbf{R},
$$

where $a, b>0, \rho>0, \quad 1<\sigma<n p /(n-p)$ if $1<p<n$ and $1<\sigma<\infty$ if $n \leq p$.

More precisely, they obtained that the global existence of solution for (1.1)-(1.2) if one of the following assumptions was satisfied:

(i) $1<\sigma<p$, the initial data $\left(u_{0}, u_{1}\right) \in W_{0}^{1, p}(\Omega) \times L^{2}(\Omega)$;

(ii) $p<\sigma$, the initial data $\left(u_{0}, u_{1}\right) \in W_{0}^{1, p}(\Omega) \times L^{2}(\Omega)$ is small.

Similar consideration can be found in [3-5]. In [6], Yang obtained the uniqueness of solution of the Laplacian wave equation (1.1)-(1.2) for $n=1$. To the best of our knowledge, there are few information on the uniqueness of solution of (1.1)-(1.2) for $n>1$ and $p>2$.

In this paper, we are interested in the global existence, the uniqueness, the continuity and the asymptotic behavior of solution for (1.1)-(1.2). The nonlinear term $g$ in (1.1) likes $g(x, u)=a(x)|u|^{\alpha-1} u-b(x)|u|^{\beta-1} u$ with $\alpha>\beta \geq 1$ and $a, b \geq 0$. Obviously, the sign condition $u g(u) \geq 0$ fails to hold for this type of function.

For these purposes, we must establish the global existence of solution for (1.1)-(1.2). Several methods have been used to study the existence of solutions to nonlinear wave equation. Notable among them is the variational approach through the use of Faedo-Galerkin approximation combined with the method of compactness and monotonicity, see [7]. To prove the uniqueness, we need to derive the various estimates for assumed solution $u(t)$. For the decay property, like (1.5), we use the method recently introduced by Martinez [8] to study the decay rate of solution to the wave equation $u_{t t}-\Delta u+g\left(u_{t}\right)=0$ in $\Omega \times \mathbf{R}^{+}$, where $\Omega$ is a bounded domain of $\mathbf{R}^{n}$.

This paper is organized as follows. In Section 2, some assumptions and the main results are stated. In Section 3, we use Faedo-Galerkin approximation together with a combination of the compactness and the monotonicity methods to prove the global existence of solution to problem (1.1)-(1.2). Further, we establish the uniqueness of solution by some a priori estimate to assumed solutions. The proof of asymptotic behavior of solution is given in Section 4. 


\section{Assumptions and Main Results}

We first give some notations and definitions. Let $\Omega$ be a bounded domain in $\mathbf{R}^{n}$ with smooth boundary $\partial \Omega$. We denote the space $L^{p}$ and $W_{0}^{1, p}$ for $L^{p}(\Omega)$ and $W_{0}^{1, p}(\Omega)$ and relevant norms by $\|\cdot\|_{p}$ and $\|\cdot\|_{1, p}$, respectively. In general, $\|\cdot\|_{X}$ denotes the norm of Banach space $X$. We also denote by $(\cdot, \cdot)$ and $\langle\cdot, \cdot\rangle\rangle$ the inner product of $L^{2}(\Omega)$ and the duality pairing between $W_{0}^{1, p}(\Omega)$ and $W^{-1, p^{\prime}}(\Omega)$, respectively. As usual, we write $u(t)$ instead $u(x, t)$. Sometimes, let $u^{\prime}(t)$ represent for $u_{t}(t)$ and so on.

If $T>0$ is given and $X$ is a Banach space, we denote by $L^{p}(0, T ; X)$ the space of functions which are $L^{p}$ over $(0, T)$ and which take their values in $X$. In this space, we consider the norm

$$
\begin{gathered}
\|u\|_{L^{p}(0, T ; X)}=\left(\int_{0}^{T}\|u(t)\|_{X}^{p} d t\right)^{1 / p}, \quad 1 \leq p<\infty, \\
\|u\|_{L^{\infty}(0, T ; X)}=\operatorname{ess} \sup _{0 \leq t \leq T}\|u(t)\|_{X} .
\end{gathered}
$$

Let us state our assumptions on $f$ and $g$.

$\left(A_{1}\right) f \in L^{p^{\prime}}$ with $p^{\prime}=p /(p-1), p>1$.

$\left(A_{2}\right)$ Let $g(x, u) \in C^{1}(\Omega \times \mathbf{R})$ and satisfy

$$
u g(x, u)+h_{1}(x)|u| \geq k_{0}\left(G(x, u)+h_{1}(x)|u|\right) \geq 0, \quad \text { in } \Omega \times \mathbf{R}
$$

and growth condition

$$
|g(x, u)| \leq k_{1}\left(|u|^{\alpha}+h_{2}(x)\right), \quad\left|g_{u}(x, u)\right| \leq k_{1}\left(|u|^{\alpha-1}+h_{3}(x)\right), \quad \text { in } \Omega \times \mathbf{R}
$$

with some $k_{0}, k_{1}>0$ and the nonnegative functions $h_{1}(x) \in L^{p^{\prime}}, h_{2} \in L^{2} \cap L^{(\alpha+1) / \alpha}, h_{3} \in$ $L^{2} \cap L^{(\alpha+1) /(\alpha-1)}$, where $1 \leq \alpha \leq n p /(n-p)-1, G(x, u)=\int_{0}^{u} g(x, s) d s$.

A typical function $g$ is $g(x, u)=a(x)|u|^{\alpha-1} u-b(x)|u|^{\beta-1} u$ with the appropriate nonnegative functions $a(x)$ and $b(x)$, where $\alpha>\beta \geq 1$.

Definition 2.1 (see [7]). A measurable function $u=u(x, t)$ on $\Omega \times \mathbf{R}^{+}$is said to be a (weak) solution of (1.1)-(1.2) if all $T>0, u \in L^{\infty}\left(0, T ; W_{0}^{1, p}\right), u_{t} \in L^{2}\left(0, T ; W_{0}^{1,2}\right), u_{t t} \in L^{2}\left(0, T ; W^{-1, p^{\prime}}\right)$, and $u$ satisfies (1.2) with $\left(u_{0}, u_{1}\right) \in W_{0}^{1, p}$ and the integral identity

$$
\int_{\Omega}\left(u_{t t} \phi+|\nabla u|^{p-2} \nabla u \cdot \nabla \phi+\nabla u_{t} \cdot \nabla \phi+g \phi-f \phi\right) d x=0
$$

for all $\phi \in C_{0}^{\infty}(\Omega)$.

Now we are in a position to state our results. 
Theorem 2.2. Assume $\left(A_{1}\right)-\left(A_{2}\right)$ hold and $\left(u_{0}, u_{1}\right) \in W_{0}^{1, p} \times L^{2}$. Then the problem (1.1)-(1.2) admits a solution $u(t)$ satisfying

$$
\begin{gathered}
u \in C\left([0, \infty) ;, W_{0}^{1,2}\right) \cap L^{\infty}\left([0, \infty) ;, W_{0}^{1, p}\right), \\
u_{t} \in L^{2}\left([0, \infty) ;, W_{0}^{1,2}\right), \quad u_{t t} \in L_{\mathrm{loc}}^{2}\left([0, \infty) ;, W^{-1, p^{\prime}}\right),
\end{gathered}
$$

and the following estimates

$$
\left\|\nabla u_{t}(t)\right\|_{2}^{2}+\|\nabla u(t)\|_{p}^{p}+\int_{0}^{t}\left\|\nabla u_{t}(s)\right\|_{2}^{2} d s \leq C_{1}(A+B), \quad \forall t \geq 0
$$

where

$$
A=\left\|u_{0}\right\|_{p}^{p}+\left\|\nabla u_{0}\right\|_{p}^{\alpha+1}+\left\|u_{1}\right\|_{2}^{2}, \quad B=H_{1}+H_{2}+H_{3}+F,
$$

with $F=\|f\|_{p^{\prime}}^{p^{\prime}}, H_{i}=\left\|h_{i}\right\|_{p^{\prime}}^{p^{\prime}}, i=1,2, H_{3}=\left\|h_{3}\right\|_{\lambda_{1}}^{\lambda_{1}}, \lambda_{1}=n / 2$.

Further, if $1 \leq \alpha \leq(n+p) /(n-p)$ and $2 \leq p \leq 4$, the solution satisfying (2.5)-(2.6) is unique.

Theorem 2.3. Let $u$ be a solution of (1.1)-(1.2) with $f=0$. In addition, let $2<p<n$ and

$$
g(x, u) u \geq p G(x, u) \geq 0, \quad \text { in } \Omega \times \mathbf{R} .
$$

Then there exists $C_{0}=C_{0}\left(u_{0}, u_{1}\right)$, such that

$$
\left\|\nabla u_{t}(t)\right\|_{2}^{2}+\|\nabla u(t)\|_{p}^{p}+\int_{\Omega} G(x, u(x, t)) d x \leq C_{0}(1+t)^{-p /(p-2)}, \quad \forall t \geq 0 .
$$

The following theorem shows that the asymptotic estimate (2.9) can be also derived if assumption (2.8) fails to hold.

Theorem 2.4. Let $u$ be a solution of (1.1)-(1.2) with $f=0$. In addition, let $2<p<n$ and

$$
g(x, u)=\lambda|u|^{\alpha-1} u-|u|^{\beta-1} u, \quad \text { in } \Omega \times \mathbf{R}
$$

with $p<\beta+1<2 p, \beta<\alpha<n p /(n-p)$. Then there exists $C_{0}=C_{0}\left(u_{0}, u_{1}\right)>0$ and $\lambda_{2}=\lambda_{2}(\alpha, \beta)>0$, such that $\lambda>\lambda_{2}$, the solution $u(t)$ satisfies

$$
\left\|\nabla u_{t}(t)\right\|_{2}^{2}+\|\nabla u(t)\|_{p}^{p}+\|u(t)\|_{\alpha+1}^{\alpha+1} \leq C_{0}(1+t)^{-p /(p-2)}, \quad \forall t \geq 0
$$




\section{Proof of Theorem 2.2}

In this section, we assume that all assumptions in Theorem 2.2 are satisfied. We first prove the global existence of a solution to problem (1.1)-(1.2) with the Faedo-Galerkin method as in $[1,2,7,9]$.

Let $r$ be an integer for which the embedding $H_{0}^{r}(\Omega)=W_{0}^{r, 2}(\Omega) \hookrightarrow W_{0}^{1, p}(\Omega)$ is continuous. Let $w_{j}(j=1,2, \ldots)$ be eigenfunctions of the spectral problem

$$
\left(w_{j}, v\right)_{H_{0}^{r}}=\lambda_{j}\left(w_{j}, v\right), \quad \forall v \in H_{0}^{r}(\Omega)
$$

where $(\cdot, \cdot)_{H_{0}^{r}}$ represents the inner product in $H_{0}^{r}(\Omega)$. Then the family $\left\{w_{1}, w_{2}, \ldots, w_{m}, \ldots\right\}$ yields a basis for both $H_{0}^{r}(\Omega)$ and $L^{2}(\Omega)$. For each integer $m$, let $V_{m}=\operatorname{span}\left\{w_{1}, w_{2}, \ldots, w_{m}\right\}$. We look for an approximate solution to problem (1.1)-(1.2) in the form

$$
u_{m}(t)=\sum_{j=1}^{m} T_{j m}(t) w_{j}
$$

where $T_{j m}(t)$ are the solution of the nonlinear ODE system in the variant $t$ :

$$
\left(u_{m}^{\prime \prime}, w_{j}\right)-\left(\Delta_{p} u_{m}, w_{j}\right)-\left(\Delta u_{m}^{\prime}, w_{j}\right)+\left(g, w_{j}\right)=\left(f, w_{j}\right), \quad j=1,2, \ldots m
$$

with the $p$-Laplacian operator $\Delta_{p} u=\operatorname{div}\left(|\nabla u|^{p-2} \nabla u\right)$ and the initial conditions

$$
u_{m}(0)=u_{0 m}, \quad u_{m}^{\prime}(0)=u_{1 m}
$$

where $u_{0 m}$ and $u_{1 m}$ are chosen in $V_{m}$ so that

$$
u_{0 m} \longrightarrow u_{0} \quad \text { in } W_{0}^{1, p}, \quad u_{1 m} \longrightarrow u_{1} \quad \text { in } L^{2}
$$

As it is well known, the system (3.3)-(3.4) has a local solution $u_{m}(t)$ on some interval $\left[0, t_{m}\right)$. We claim that for any $T>0$, such a solution can be extended to the whole interval $[0, T]$ by using the first a priori estimate below. We denote by $C_{k}$ the constant which is independent of $m$ and the initial data $u_{0}$ and $u_{1}$.

Multiplying (3.3) by $T_{j m}^{\prime}(t)$ and summing the resulting equations over $j$, we get after integration by parts

$$
E_{m}^{\prime}(t)+\left\|\nabla u_{m}^{\prime}(t)\right\|_{2}^{2}=0, \quad \forall t \geq 0,
$$

where

$$
E_{m}(t)=\frac{1}{2}\left\|u_{m}^{\prime}(t)\right\|_{2}^{2}+\frac{1}{p}\left\|\nabla u_{m}(t)\right\|_{p}^{p}+\int_{\Omega} G\left(x, u_{m}\right) d x-\int_{\Omega} f(x) u_{m} d x
$$


By (2.2) and Young inequality, we have

$$
\begin{gathered}
\int_{\Omega} G\left(x, u_{m}\right) d x \geq-\int_{\Omega} h_{1}(x)\left|u_{m}\right| d x \geq-\varepsilon\left\|\nabla u_{m}\right\|_{p}^{p}-C_{\varepsilon}\left\|h_{1}\right\|_{p^{\prime}}^{p^{\prime}} \\
\int_{\Omega} f(x) u_{m} d x \geq-\varepsilon\left\|\nabla u_{m}\right\|_{p}^{p}-C_{\varepsilon}\|f\|_{p^{\prime}}^{p^{\prime}}
\end{gathered}
$$

Let $\varepsilon>0$ be so small that $2 p^{-1}-4 \varepsilon \geq p^{-1}$. Then

$$
E_{m}(t) \geq \frac{1}{2}\left\|u_{m}^{\prime}(t)\right\|_{2}^{2}+\frac{1}{2 p}\left\|\nabla u_{m}(t)\right\|_{p}^{p}-C_{1}\left(H_{1}+F\right),
$$

or

$$
\left\|u_{m}^{\prime}(t)\right\|_{2}^{2}+\left\|\nabla u_{m}(t)\right\|_{p}^{p} \leq C_{1}\left(E_{m}(t)+H_{1}+F_{1}\right)
$$

for some $C_{1}>0$.

Thus, it follows from (3.6) and (3.10) that, for any $m=1,2, \ldots$, and $t \geq 0$

$$
\left\|u_{m}^{\prime}(t)\right\|_{2}^{2}+\left\|\nabla u_{m}(t)\right\|_{p}^{p}+\int_{0}^{t}\left\|\nabla u_{m}(s)\right\|_{2}^{2} d s \leq C_{2}\left(E_{m}(0)+H_{1}+F_{1}\right) .
$$

By assumption $\left(A_{2}\right)$, we obtain that $\alpha+1 \leq n p /(n-p)$ and

$$
\begin{aligned}
\left|\int_{\Omega} G\left(x, u_{m}\right) d x\right| & \leq k_{1}\left(\left\|u_{m}\right\|_{\alpha+1}^{\alpha+1}+\int_{\Omega}\left|h_{2} \| u_{m}\right| d x\right) \\
& \leq C_{2}\left(\left\|\nabla u_{m}\right\|_{p}^{\alpha+1}+\left\|u_{m}\right\|_{p}^{p}+\left\|h_{2}\right\|_{p^{\prime}}^{p^{\prime}}\right) \\
& \leq C_{2}\left(\left\|\nabla u_{m}\right\|_{p}^{\alpha+1}+\left\|\nabla u_{m}\right\|_{p}^{p}+H_{2}\right) .
\end{aligned}
$$

Then it follows (3.5) and (3.6) that

$$
\begin{aligned}
E_{m}(t) \leq E_{m}(0) & =\frac{1}{2}\left\|u_{1 m}^{\prime}\right\|_{2}^{2}+\frac{1}{p}\left\|\nabla u_{0 m}\right\|_{p}^{p}+\int_{\Omega} G\left(x, u_{0 m}\right) d x-\int_{\Omega} f(x) u_{0 m} d x \\
& \leq C_{2}\left(\left\|u_{1}\right\|_{2}^{2}+\left\|\nabla u_{0}\right\|_{p}^{p}+\left\|\nabla u_{0}\right\|_{p}^{\alpha}+H_{1}+H_{2}+F\right) \\
& \leq C_{2}(A+B) .
\end{aligned}
$$

Hence, for any $t \geq 0$ and $m=1,2, \ldots$, we have from (3.11) and (3.13) that

$$
\left\|u_{m}^{\prime}(t)\right\|_{2}^{2}+\left\|\nabla u_{m}(t)\right\|_{p}^{p}+\int_{0}^{t}\left\|\nabla u_{m}^{\prime}(s)\right\|_{2}^{2} d s \leq C_{2}(A+B), \quad \forall t \geq 0 .
$$


With this estimate we can extend the approximate solution $u_{m}(t)$ to the interval $[0, T]$ and we have that

$$
\begin{gathered}
\left\{u_{m}(t)\right\} \text { is bounded in } L^{\infty}\left(0, T ; W_{0}^{1, p}\right), \\
\left\{u_{m}^{\prime}(t)\right\} \text { is bounded in } L^{\infty}\left(0, T ; L^{2}\right), \\
\left\{u_{m}^{\prime}(t)\right\} \text { is bounded in } L^{2}\left(0, T ; W_{0}^{1,2}\right) .
\end{gathered}
$$

Now we recall that operator $-\Delta_{p} u=-\operatorname{div}\left(|\nabla u|^{p-2} \nabla u\right)$ is bounded, monotone, and hemicontinuous from $W_{0}^{1, p}$ to $W^{-1, p^{\prime}}$ with $p \geq 2$. Then we have

$$
\left\{-\Delta_{p} u_{m}(t)\right\} \text { is bounded } L^{\infty}\left(0, T ; W^{-1, p^{\prime}}\right) .
$$

By the standard projection argument as in [1], we can get from the approximate equation (3.3) and the estimates (3.15)-(3.17) that

$$
\left\{u_{m}^{\prime \prime}(t)\right\} \text { is bounded in } L^{2}\left(0, T ; \mathrm{H}^{-r}(\Omega)\right) \text {. }
$$

From (3.15)-(3.16), going to a subsequence if necessary, there exists $u$ such that

$$
\begin{gathered}
u_{m} \rightarrow u \text { weakly star in } L^{\infty}\left(0, T ; W_{0}^{1, p}\right), \\
u_{m}^{\prime} \rightarrow u^{\prime} \text { weakly star in } L^{\infty}\left(0, T ; L^{2}\right), \\
u_{m}^{\prime} \rightarrow u^{\prime} \text { weakly in } L^{2}\left(0, T ; L^{2}\right),
\end{gathered}
$$

and in view of (3.18), there exists $\chi(t)$ such that

$$
-\Delta_{p} u_{m}(t) \rightarrow \chi(t) \text { weakly star in } L^{\infty}\left(0, T ; W^{-1, p^{\prime}}\right)
$$
(3.16),

By applying the Lions-Aubin compactness Lemma in [7], we get, from (3.15) and

$$
u_{m} \longrightarrow u \text { strongly in } L^{2}\left(0, T ; L^{2}\right)
$$

and $u_{m} \rightarrow u$ a.e. in $\Omega \times(0, T)$. 
Since the embedding $W_{0}^{1,2} \hookrightarrow L^{2}$ is compact, we get, from (3.18) and (3.19),

$$
u_{m}^{\prime} \longrightarrow u^{\prime} \text { strongly in } L^{2}\left(0, T ; L^{2}\right)
$$

Using the growth condition (2.3) and (3.25), we see that

$$
\int_{0}^{T} \int_{\Omega}\left|g\left(x, u_{m}(x, t)\right)\right|^{(\alpha+1) / \alpha} d x d t
$$

is bounded and

$$
g\left(x, u_{m}\right) \longrightarrow g(x, u) \text { a.e. in }(\Omega \times T)
$$

Therefore, from [7, Chapter 1, Lemma 1.3], we infer that

$$
g\left(x, u_{m}\right) \rightarrow g(x, u) \text { weakly in } L^{(\alpha+1) / \alpha}\left(0, T ; L^{(\alpha+1) / \alpha}\right) .
$$

With these convergences, we can pass to the limit in the approximate equation and then

$$
\frac{d}{d t}\left(u^{\prime}(t), v\right)+\langle\chi(t), v\rangle+\left(\nabla u^{\prime}, \nabla v\right)+(g, v)=(f, v), \quad \forall v \in W_{0}^{1, p}
$$

Obviously, $u$ satisfies the estimates (2.5)-(2.6). Finally, using the standard monotonicity argument as done in $[1,7]$, we get that $\chi(t)=-\Delta_{p} u(t)$. This completes the proof of existence of solution $u(t)$.

To prove the uniqueness, we assume that $u(t)$ and $v(t)$ are two solutions which satisfy (2.5)-(2.6) and $u(0)=v(0), u_{t}(0)=v_{t}(0)$. Setting $U(t)=u_{t}(t), V(t)=v_{t}(t)$, and $W(t)=$ $U(t)-V(t)$. We see from (1.1) and (1.2) that

$$
W_{t}-\Delta W-\operatorname{div}\left(|\nabla u|^{p-2} \nabla u-|\nabla v|^{p-2} \nabla v\right)=g(x, v)-g(x, u) .
$$

Multiplying (3.30) by $W$ and integrating over $\Omega$, we have

$$
\begin{aligned}
& \frac{1}{2} \frac{d}{d t}\|W(t)\|_{2}^{2}+\|\nabla W(t)\|_{2}^{2}+\int_{\Omega}\left(|\nabla u|^{p-2} \nabla u-|\nabla v|^{p-2} \nabla v\right) \nabla W d x=\int_{\Omega}(g(x, v)-g(x, u)) W d x, \\
& \|W(t)\|_{2}^{2}+2 \int_{0}^{t}\|\nabla W(s)\|_{2}^{2} d s+2 \int_{0}^{t} \int_{\Omega}\left(|\nabla u|^{p-2} \nabla u-|\nabla v|^{p-2} \nabla v\right) \nabla W d x d \tau \\
& =2 \int_{0}^{t} \int_{\Omega}(g(x, v)-g(x, u)) W d x d s
\end{aligned}
$$


Journal of Inequalities and Applications

Now setting $U_{\epsilon}=\epsilon u+(1-\epsilon) v, 0 \leq \epsilon \leq 1$, then

$$
\begin{aligned}
& \int_{0}^{t} \int_{\Omega}\left|\left(|\nabla u|^{p-2} \nabla u-|\nabla v|^{p-2} \nabla v\right) \nabla W\right| d x d \tau \\
& \quad \leq \int_{0}^{t} \int_{\Omega}\left|\int_{0}^{1} \frac{d}{d \epsilon}\left(\left|\nabla U_{\epsilon}\right|^{p-2} \nabla U_{\epsilon}\right) d \epsilon\right||\nabla W| d x d \tau \\
& \quad \leq(p-1) \int_{0}^{t} \int_{\Omega} \int_{0}^{1}\left|\nabla U_{\epsilon}\right|^{p-2}|\nabla(u(\tau)-v(\tau))||\nabla W| d \epsilon d x d \tau \equiv I .
\end{aligned}
$$

Note that

$$
\begin{gathered}
\left|\nabla U_{\epsilon}(\tau)\right| \leq|\nabla u(\tau)|+|\nabla v(\tau)| \\
|\nabla(u(\tau)-v(\tau))| \leq \int_{0}^{\tau}\left|\nabla\left(u_{s}(s)-v_{s}(s)\right)\right| d s=\int_{0}^{\tau}|\nabla W(s)| d s .
\end{gathered}
$$

Then, by the estimates (2.6) and $2 \leq p \leq 4$, we have

$$
\begin{aligned}
I & \leq C_{1} \int_{0}^{t} \int_{\Omega} \int_{0}^{\tau}\left(|\nabla u(\tau)|^{p-2}+|\nabla v(\tau)|^{p-2}\right)|\nabla W(s) \| \nabla W(\tau)| d x d s d \tau \\
& \leq C_{1} \int_{0}^{t} \int_{0}^{\tau}\left(\|\nabla u(\tau)\|_{p}^{p-2}+\|\nabla v(\tau)\|_{p}^{p-2}\right)\|\nabla W(s)\|_{2}\|\nabla W(\tau)\|_{2} d s d \tau \\
& \leq C_{1}(A+B)^{(p-2) / p} \int_{0}^{t} \int_{0}^{\tau}\|\nabla W(s)\|_{2}\|\nabla W(\tau)\|_{2} d s d \tau \\
& \leq C_{1}(A+B)^{(p-2) / p}\left(\int_{0}^{t}\|\nabla W(s)\|_{2} d s\right)^{2} \leq C_{2} t \int_{0}^{t}\|\nabla W(s)\|_{2}^{2} d s
\end{aligned}
$$

with $C_{2}=C_{1}(A+B)^{(p-2) / p}$.

For the term of the right side to (3.31), we have

$$
\begin{aligned}
G_{1} & =\int_{0}^{t} \int_{\Omega}|g(x, v)-g(x, u)||W| d x d \tau=\int_{0}^{t} \int_{\Omega}\left|\int_{0}^{1} \frac{d}{d \epsilon} g\left(x, U_{\epsilon}\right) d \epsilon\right||W| d x d \tau \\
& \leq \int_{0}^{t} \int_{\Omega} \int_{0}^{1}\left|g_{u}\left(x, U_{\epsilon}\right)\|u(\tau)-v(\tau)\| W(\tau)\right| d \epsilon d x d \tau \\
& \leq \int_{0}^{t} \int_{0}^{\tau} \int_{0}^{1}\left\|g_{u}\left(x, U_{\epsilon}\right)\right\|_{\lambda_{1}} d \epsilon\left\|u_{s}(s)-v_{s}(s)\right\|_{\lambda_{2}}\|W(\tau)\|_{\lambda_{2}} d \epsilon d s d \tau
\end{aligned}
$$

with $\lambda_{1}=n / 2, \lambda_{2}=2 n /(n-2)$. 
By the assumption $\left(A_{2}\right)$ and $1 \leq \alpha \leq(n+p) /(n-p)$, we see that

$$
\begin{aligned}
\left\|g_{u}\left(x, U_{\epsilon}\right)\right\|_{\lambda_{1}}^{\lambda_{1}} & \leq k_{1} \int_{\Omega}\left(|u(\tau)|^{\alpha-1}+|v(\tau)|^{\alpha-1}+\left|h_{3}\right|\right)^{n / 2} d x \\
& \leq C_{3} \int_{\Omega}\left(|u(\tau)|^{n(\alpha-1) / 2}+|v(\tau)|^{n(\alpha-1) / 2}+\left|h_{3}\right|^{n / 2}\right) d x \\
& \leq C_{3}\left(\|\nabla u(\tau)\|_{\mathrm{p}}^{n(\alpha-1) / 2}+\|\nabla v(\tau)\|_{p}^{n(\alpha-1) / 2}+H_{3}\right) .
\end{aligned}
$$

By the estimate (2.6), we have

$$
\|\nabla u(t)\|_{p}, \quad\|v(t)\|_{p} \leq C_{2}(A+B)^{1 / p}, \quad \forall t \geq 0
$$

Therefore, there exists $C_{4}>0$, depending $u_{0}, v_{0}, f, h_{i}$ such that

$$
\left\|g_{u}\left(x, U_{\epsilon}\right)\right\|_{\lambda_{1}} \leq C_{4}, \quad \forall t \geq 0
$$

Since $u, v \in W_{0}^{1, p} \subset W_{0}^{1,2}, u_{t}, v_{t} \in W_{0}^{1,2}$, we get

$$
\begin{gathered}
\left\|u_{s}(s)-v_{s}(s)\right\|_{\mathcal{\lambda}_{2}} \leq C_{0}\left\|\nabla\left(u_{s}(s)-v_{s}(s)\right)\right\|_{2}=C_{0}\|\nabla W(s)\|_{2}, \\
\|W(\tau)\|_{2} \leq C_{0}\|\nabla W(\tau)\|_{2} .
\end{gathered}
$$

Then (3.35) becomes

$$
G_{1} \leq C_{4} \int_{0}^{t} \int_{0}^{\tau}\|W(s)\|_{\lambda_{2}}\|W(\tau)\|_{\lambda_{2}} d s d \tau \leq C_{4}\left(\int_{0}^{t}\|\nabla W(s)\|_{2} d s\right)^{2} \leq C_{4} t \int_{0}^{t}\|\nabla W(s)\|_{2}^{2} d s .
$$

Therefore, it follows from (3.31), (3.34), and (3.40) that

$$
\|W(t)\|_{2}^{2}+2 \int_{0}^{t}\|\nabla W(s)\|_{2}^{2} d s \leq\left(C_{2}+C_{4}\right) t \int_{0}^{t}\|\nabla W(s)\|_{2}^{2}
$$

The integral inequality (3.41) shows that there exists $T_{1}>0$, such that

$$
W(t)=0, \quad 0 \leq t \leq T_{1} .
$$

Consequently, $u(t)-v(t)=u(0)-v(0)=0,0 \leq t \leq T_{1}$. 
Repeating the above procedure, we conduce that $u(t)=v(t)$ on $\left[T_{1}, 2 T_{1}\right],\left[2 T_{1}, 3 T_{1}\right], \ldots$ and $u(t)=v(t)$ on $[0, \infty)$. This ends the proof of uniqueness.

Next, we prove that $u \in C\left([0, \infty) ; W_{0}^{1,2}\right)$. Let $t>s \geq 0$, we have

$$
\begin{aligned}
\|\nabla(u(t)-u(s))\|_{2}^{2} & =\int_{\Omega}\left|\int_{s}^{t} \nabla u_{\tau}(\tau) d \tau\right|^{2} d x \leq \int_{\Omega} \int_{s}^{t}\left|\nabla u_{\tau}(\tau)\right|^{2} d s d x(t-s) \\
& =(t-s) \int_{s}^{t}\left\|\nabla u_{\tau}(\tau)\right\|_{2}^{2} d \tau \longrightarrow 0, \quad \text { as } t \longrightarrow s .
\end{aligned}
$$

This shows that $u(t) \in C\left([0, \infty) ; W_{0}^{1,2}\right)$. We complete the proof of Theorem 2.2.

\section{Proof of Theorem 2.3}

Let us first state a well-known lemma that will be needed later.

Lemma 4.1 (see [10]). Let $E: \mathbf{R}^{+} \rightarrow \mathbf{R}^{+}$be a nonincreasing function and assume that there are constants $q \geq 0$ and $r>0$, such that

$$
\int_{S}^{\infty} E^{q+1}(t) d t \leq \gamma^{-1} E^{q}(0) E(S), \quad \forall S \geq 0
$$

Then, we have

$$
\begin{gathered}
E(t) \leq E(0)\left(\frac{1+q}{1+q \gamma t}\right)^{1 / q}, \quad \forall t \geq 0, \text { if } q>0, \\
E(t) \leq E(0) e^{1-\gamma t}, \quad \forall t \geq 0, \text { if } q=0 .
\end{gathered}
$$

\subsection{The Proof of Theorem 2.3}

Let

$$
E(t)=\frac{1}{2}\left\|u_{t}(t)\right\|_{2}^{2}+\frac{1}{p}\|\nabla u(t)\|_{p}^{p}+\int_{\Omega} G(x, u) d x, \quad t \geq 0
$$

Then, we have from (1.1) that

$$
E^{\prime}(t)+\left\|\nabla u_{t}(t)\right\|_{2}^{2}=0, \quad \forall t \geq 0
$$

This shows that $E(t)$ is nonincreasing in $[0, \infty)$. 
Multiplying (1.1) by $E^{q}(\mathrm{t}) u(t)$ with $q=(p-2) / p>0$, we get

$$
\int_{S}^{T} E^{q}(t) \int_{\Omega} u\left(u_{t t}-\Delta_{p} u-\Delta u_{t}+g(x, u)\right) d x d t=0, \quad \forall T>S \geq 0 .
$$

Note that

$$
\begin{aligned}
\int_{S}^{T} E^{q}(t)\left(u, u_{t t}\right) d t= & \left.E^{q}(t)\left(u, u_{t}\right)\right|_{S} ^{T}-\int_{S}^{T}\left(q E^{q-1}(t) E^{\prime}(t)\left(u, u_{t}\right)+E^{q}(t)\left\|u_{t}(t)\right\|_{2}^{2}\right) d t \\
& -\int_{S}^{T} E^{q}(t)\left(u, \Delta_{p} u\right) d t=\int_{S}^{T} E^{q}(t)\|\nabla u(t)\|_{p}^{p} d t \\
& -\int_{S}^{T} E^{q}(t)\left(u, \Delta u_{t}\right) d t=\int_{S}^{T} E^{q}(t)\left(\nabla u, \nabla u_{t}\right) d t .
\end{aligned}
$$

Then we have from (4.5) that

$$
\begin{aligned}
p \int_{S}^{T} E^{q+1}(t) d t= & -\left.E^{\mathrm{q}}(t)\left(u, u_{t}\right)\right|_{S} ^{T}+q \int_{S}^{T} E^{q-1}(t) E^{\prime}(t)\left(u, u_{t}\right) d t \\
& +\left(1+\frac{p}{2}\right) \int_{S}^{T} E^{q}(t)\left\|u_{t}(t)\right\|_{2}^{2} d t-\int_{S}^{T} E^{q}(t)\left(\nabla u, \nabla u_{t}\right) d t \\
& +\int_{S}^{T} E^{q}(t) \int_{\Omega}(p G(u)-u g(u) d x d t .
\end{aligned}
$$

Since $\int_{\Omega} G(x, u) d x \geq 0, E(t) \geq 0$. Further, by (4.4), we see that

$$
\begin{gathered}
\left\|\nabla u_{t}(t)\right\|_{2} \leq\left(-E^{\prime}(t)\right)^{1 / 2}, \quad\|\nabla u(t)\|_{p} \leq p E^{1 / p}(t), \quad \forall t \geq 0, \\
\left|E^{q}(t)\left(u, u_{t}\right)\right| \leq E^{q}(t)\|u(t)\|_{2}\left\|u_{t}(t)\right\|_{2} \leq C_{0} E^{q}(t)\|\nabla u(t)\|_{p}\left\|\nabla u_{t}(t)\right\|_{2} \leq C_{0}(E(t))^{\mu_{1}}
\end{gathered}
$$

with $\mu_{1}=q+1 / 2+1 / p$.

This gives

$$
\left.E^{q}(t)\left(u, u_{t}\right)\right|_{S} ^{T} \leq C_{1} E^{\mu_{1}}(S), \quad \forall T>S \geq 0,
$$

where the fact that $E(t)$ is nonincreasing is used. 
Journal of Inequalities and Applications

Similarly, we derive the following estimates

$$
\begin{aligned}
\int_{S}^{T} E^{q}(t)\left\|u_{t}(t)\right\|_{2}^{2} d t & \leq C_{1} \int_{S}^{T} E^{q}(t)\left\|\nabla u_{t}(t)\right\|_{2}^{2} d t \\
& =C_{1} \int_{S}^{T} E^{q}(t)\left(-E^{\prime}(t)\right) d t \leq C_{1} E^{q+1}(S), \\
q \int_{S}^{T}\left|E^{q-1}(t) E^{\prime}(t)\left(u, u_{t}\right)\right| d t & \leq C_{1} \int_{S}^{T} E^{q-1}(t)\left|E^{\prime}(t)\right|\|u(t)\|_{2}\left\|u_{t}(t)\right\|_{2} d t \\
& \leq C_{1} \int_{S}^{T} E^{\mu_{1}-1}(t)\left|E^{\prime}(t)\right| d t \leq C_{1} E^{\mu_{1}}(S), \\
\int_{S}^{T}\left|E^{q}(t)\left(\nabla u, \nabla u_{t}\right)\right| d t & \leq \int_{S}^{T} E^{q}(t)\|\nabla u(t)\|_{2}\left\|\nabla u_{t}(t)\right\|_{2} d t \\
& \leq C_{1} \int_{S}^{T} E^{q+1 / p}(t)\left(-E^{\prime}(t)\right)^{1 / 2} d t \\
& \leq \int_{S}^{T} E^{q+1}(t) d t+C_{1} \int_{S}^{T} E^{q+2 / p-1}(t)\left(-E^{\prime}(t)\right) d t \\
& \leq \int_{S}^{T} E^{q+1}(t) d t+C_{1} E^{q+2 / p}(S) .
\end{aligned}
$$

Then we get from (4.9)-(4.12) that

$$
\begin{aligned}
\int_{S}^{T} E^{q+1}(t) d t & \leq C_{1}\left(E^{\mu_{1}}(S)+E^{q+1}(S)+E^{q+2 / p}(S)\right) \\
& \leq C_{1} E(S)\left(E^{\mu_{1}}(S)+E^{q}(S)+E^{q+2 / p-1}(S)\right) \\
& \leq C_{1} E(S) E^{q}(0)\left(E^{1 / p-1 / 2}(0)+1+E^{2 / p-1}(0)\right) \\
& \equiv r^{-1} E^{q}(0) E(S),
\end{aligned}
$$

for any $T>S \geq 0$, letting $T \rightarrow \infty$, we find that

$$
\int_{S}^{\infty} E^{q+1}(t) d t \leq r^{-1} E(S) E^{q}(0), \quad \forall S \geq 0
$$

By Lemma 4.1, we obtain that

$$
E(t)=\frac{1}{2}\left\|u_{t}(t)\right\|_{2}^{2}+\frac{1}{p}\|\nabla u(t)\|_{p}^{p}+\int_{\Omega} G(x, u) d x \leq E(0)\left(\frac{1+q}{1+q \gamma t}\right)^{1 / q} \leq C_{2} E(0)(1+t)^{-p /(p-2)} .
$$

This is (2.9) and we complete the proof of Theorem 2.3. 


\subsection{The Proof of Theorem 2.4}

By Sobolev inequality, we know that there exists $\lambda_{0}>0$ such that

$$
\lambda_{0}\|u\|_{p}^{p} \leq\|\nabla u\|_{p}^{p}, \quad \forall u \in W_{0}^{1, p}(\Omega) .
$$

Let $u$ be a solution for (1.1)-(1.2) in Theorem 2.2. By (2.10),

$$
G(u)=\frac{\lambda}{\alpha+1}|u|^{\alpha+1}-\frac{1}{\beta+1}|u|^{\beta+1} .
$$

Obviously, there exists $\lambda_{2}>0$, such that $\lambda>\lambda_{2}$,

$$
\frac{\lambda_{0}}{2 p}|u|^{p}+G(u) \geq \frac{1}{2(\alpha+1)}|u|^{\alpha+1}, \quad \forall u \in \mathbf{R} .
$$

This implies that

$$
\begin{gathered}
\frac{\lambda_{0}}{2 p}\|u\|_{p}^{p}+\int_{\Omega} G(u) d x \geq \frac{1}{2(\alpha+1)}\|u\|_{\alpha+1}^{\alpha+1}, \\
E(t) \geq \frac{1}{2}\left\|u_{t}(t)\right\|_{2}^{2}+\frac{1}{2 p}\|\nabla u(t)\|_{p}^{p}+\frac{1}{2(\alpha+1)}\|u(t)\|_{\alpha+1}^{\alpha+1} .
\end{gathered}
$$

On the other hand, we have, from (4.18) and (4.19),

$$
\begin{aligned}
p G(u)-u g(u) & =\frac{\beta+1-p}{\beta+1}|u|^{\beta+1}-\frac{\lambda(\alpha+1-p)}{\alpha+1}|u|^{\alpha+1} \\
& \leq \frac{\beta+1-p}{\beta+1}|u|^{\beta+1}=(\beta+1-p)\left(\frac{\lambda}{\alpha+1}|u|^{\alpha+1}-G(u)\right) \\
& \leq(\beta+1-p)\left(\frac{\lambda_{0}}{p}|u|^{p}+G(u)\right) .
\end{aligned}
$$

It shows that

$$
\int_{S}^{T} E^{q}(t) \int_{\Omega}(p G(u)-g u) d x d t \leq(\beta+1-p) \int_{S}^{T} E^{q+1}(t) d t .
$$

Then, by (4.9) and (4.11)-(4.14), we have

$$
\begin{aligned}
(2 p-\beta-1) \int_{S}^{T} E^{q+1}(t) d t & \leq C_{0}\left(E^{q+1 / p+2}(S)+E^{q+1}(S)+E^{q+2 / p}(S)\right) \\
& \leq \gamma^{-1} E(S) E^{q}(0) .
\end{aligned}
$$


The applications of Lemma 4.1 and (4.19) yields that

$$
\left\|u_{t}(t)\right\|_{2}^{2}+\|\nabla u(t)\|_{2}^{2}+\|u(t)\|_{\alpha+1}^{\alpha+1} \leq C_{0}(1+t)^{-p /(p-2)}, \quad \forall t \geq 0 .
$$

This ends the proof of Theorem 2.4.

\section{Acknowledgments}

The authors express their sincere gratitude to the anonymous referees for a number of valuable comments and suggestions. The work was supported by the Science Funds of Hohai University (Grant Nos. 2008430211 and 2008408306) and the Fundamental Research Funds for the Central Universities (Grant No. 2010B17914).

\section{References}

[1] T. F. Ma and J. A. Soriano, "On weak solutions for an evolution equation with exponential nonlinearities," Nonlinear Analysis: Theory, Methods E Applications, vol. 37, no. 8, pp. 1029-1038, 1999.

[2] H. Gao and T. F. Ma, "Global solutions for a nonlinear wave equation with the $p$-Laplacian operator," Electronic Journal of Qualitative Theory of Differential Equations, no. 11, pp. 1-13, 1999.

[3] A. Benaissa and A. Guesmia, "Energy decay for wave equations of $\phi$-Laplacian type with weakly nonlinear dissipation," Electronic Journal of Differential Equations, vol. 2008, no. 109, pp. 1-22, 2008.

[4] A. C. Biazutti, "On a nonlinear evolution equation and its applications," Nonlinear Analysis: Theory, Methods \& Applications, vol. 24, no. 8, pp. 1221-1234, 1995.

[5] M. Nakao and Z. J. Yang, "Global attractors for some quasi-linear wave equations with a strong dissipation," Advances in Mathematical Sciences and Applications, vol. 17, no. 1, pp. 89-105, 2007.

[6] Z. J. Yang, "Existence and asymptotic behaviour of solutions for a class of quasi-linear evolution equations with non-linear damping and source terms," Mathematical Methods in the Applied Sciences, vol. 25, no. 10, pp. 795-814, 2002.

[7] J.-L. Lions, Quelques Méthodes de Résolution des Problèmes aux Limites non Linéaires, Dunod-Gauthier Villars, Paris, France, 1969.

[8] P. Martinez, "A new method to obtain decay rate estimates for dissipative systems," ESAIM: Control, Optimisation and Calculus of Variations, vol. 4, pp. 419-444, 1999.

[9] M. Sango, "On a nonlinear hyperbolic equation with anisotropy: global existence and decay of solution," Nonlinear Analysis: Theory, Methods \& Applications, vol. 70, no. 7, pp. 2816-2823, 2009.

[10] V. Komornik, Exact Controllability and Stabilization: The Multiplier Method, RAM: Research in Applied Mathematics, John Wiley \& Sons, Chichester, UK; Masson, Paris, France, 1994. 